\title{
The Oval Pupil
}

\author{
H. S. DUA. N. J. WATSON, J. V. FORRESTER \\ Aberdeen
}

\begin{abstract}
Summary
The dynamics of pupillary dilation induced by Phenylephrine $10 \%$ and Cyclopentolate $1 \%$ have been examined by flash photography. A correlation between anterior chamber depth and the pupil shape on dilation with Phenylephrine Hydrochloride $10 \%$ is described. It is postulated that these pupillary dilation dynamics support a sympathetic abnormality as a trigger for acute primary angle closure glaucoma.
\end{abstract}

The oval pupil is a common clinical sign in acute primary angle closure glaucoma (APACG).' APACG has been shown to occur more commonly in patients with shallow anterior chambers. - The acute attack is precipitated by autonomic dysfunction and a modification of physiological pupil block. During an attack of APACG the pupil is middilated and vertically elongated, a preferential dilation in the vertical meridian producing the oval shape." Oval or 'tadpole shaped" pupils have also been reported in patients with Horner's syndrome. ${ }^{+}$As both these conditions with oval pupils have an underlying sympathetic dysfunction, a cause and effect relationship may be inferred. We undertook this study to examine this relationship further by comparing the dilation characteristics of Phenylephrine Hydrochloride (a direct acting amine) and Cyclopentolate Hydrochloride (a parasympathetic agonist). Particular attention was paid to any differential dilation in the vertical meridian.

\section{Subjects and methods}

Informed consent was obtained from 43 subjects ( 86 eyes). There were 17 males and 26 females between 15 and 89 years of age. Recruitment was from general ophthalmic clinics after ocular and general examination had excluded conditions known to affect ocular sympathetic function. Exclusion criteria included diabetes mellitus, glaucoma or ocular hypertension, previous ocular surgery (including extraocular muscle surgery). previous intraocular inflammation and topical drug administration. The colour of the iris was noted and the anterior chamber depth was measured on a Haag Streit slit lamp using the technique described by Smith." A fine beam is directed horizontally at the eye from $60^{\circ}$. Its length is adjusted until the corneal and iridal reflexes meet. The length of the beam required is proportional to the depth of the anterior chamber $(1.117 \times$ slit beam length $+0.5(079)$.

Twenty-eight eyes had anterior chamber depths of less than $2.2 \mathrm{~mm}$ and the remaining 58 eyes had anterior chamber depths of greater than $2.2 \mathrm{~mm}$. Details of the study group are shown in Table I. Two drops of Phenylephrine hydrochloride $10 \%$ were instilled into the inferior fornix of one eye and Cyclopentolate $1 \%$ into the second eye of each subject. The right and left eyes were randomly selected for each of these drugs. Pupillary size was recorded, in dim background illumination, by flash photography at $0,5,10,15,20$

Correspondence to: N. J. Watson, Department of Ophthalmology, University of Aberdeen, Foresterhill, Aberdeen AB9 2ZB. 
Table I Subject characteristics

Age range $15-89$ years

Sex

Male 17 patients

Female 26 patients

Chamber depth

Anterior chamber $<2.2 \mathrm{~mm} 28$ eyes

Anterior chamber $>2.2 \mathrm{~mm} 58$ eyes

Iris colour

Light 48 eyes

Dark 38 eyes

and 25 minutes. In order to eliminate the problems of magnification the pupil dilation was recorded as the ratio of pupil size vertically and horizontally, a vertically oval pupil having a pupil dilation ratio $>1$ (see Fig. 1).

The intraocular pressure was monitored throughout the procedure and pupillary dilation reversed with Pilocarpine hydrochloride $4 \%$ either after 25 minutes or if there was an increase in intraocular pressure. Subjects who showed a rise in intraocular pressure over $30 \mathrm{~mm}$ of mercury were excluded from the study, and referred for further ophthalmological care.

\section{Results}

Eyes treated with Cyclopentolate hydrochlo-

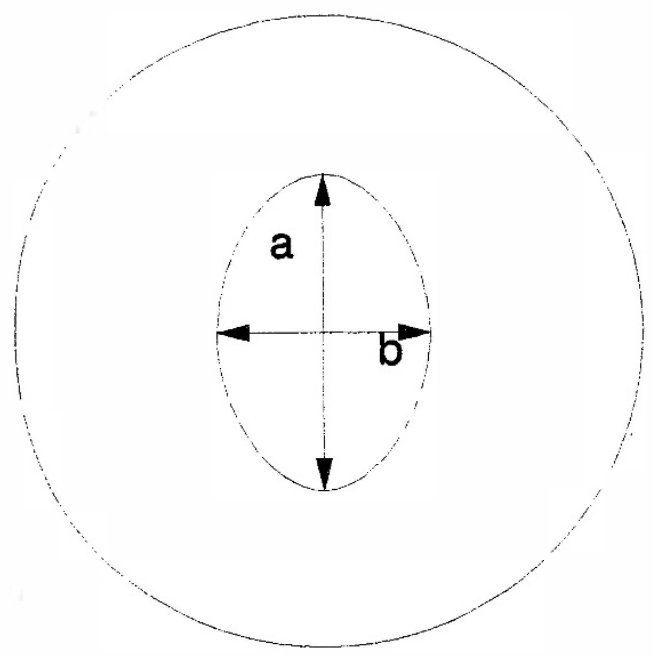

\section{Dilation Ratio $=\mathrm{a} / \mathrm{b}$}

Fig. 1. The dilation ratio is defined as the ratio of pupil size vertically to horizontally: This eliminates problems of magnification. ride $1 \%$ did not show any meridional preference during dilation. Twenty-seven of the 43 eyes treated with Phenylephrine hydrochloride $10 \%$ showed a preferential dilation in the vertical meridian, the pupils becoming vertically oval, prior to resuming a round contour on complete mydriasis (Figs. 2 and 3). A maximum dilation ratio was obtained 15 to 20 minutes after drug instillation. The time point at which maximal vertical dilation occurred with Phenylephrine hydrochloride $10 \%$ had no relationship to the anterior chamber depth. The 15 minute dilation ratios of the right and left eyes of each subject were compared. The differences in dilation ratio between Cyclopentolate and Phenylephrine treated eyes was statistically significant $(\mathrm{P}<0.05$ chi sq test $)$ in subjects with anterior chamber depths less than $2.2 \mathrm{~mm}$ (Figs. 4 and 5). A significant direct relationship was found between anterior chamber depth and dilation ratio, the shallower the anterior chamber, the greater the dilation ratio $(r=0.526$, Fig. 6$)$. Other factors like iris colour, age and sex had no relation to the dilation ratio.

\section{Discussion}

The results of this study indicate that Cyclopentolate hydrochloride $1 \%$ and Phenylephrine hydrochloride $10 \%$ have different pupillary dilation dynamics. This has not been previously described. These drugs act on different components of the autonomic nervous system suggesting that differential innervation is responsible for the oval pupil. A reduction in the autonomic responses with age has been reported both for systemic and

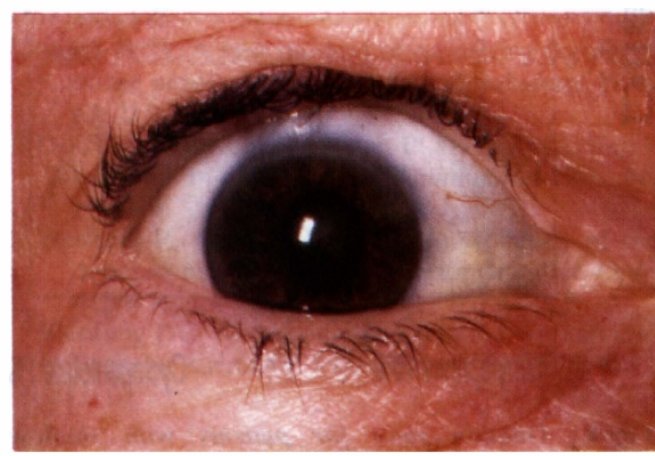

Fig. 2. Ele treated with Phenylephrine Hydrochloride $10 \%$ demonstrates preferential dilation in the vertical meridian. Photographs at 0 and 15 minutes. 


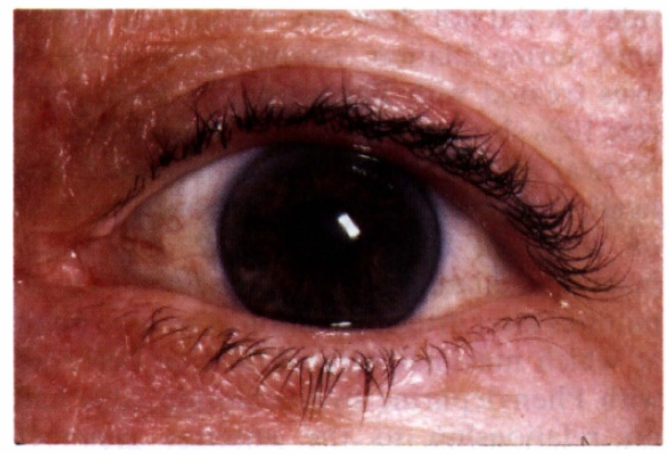

Fig. 3. Eye treated with Cyclopentolate Hydrochloride $1 \%$ does not show preferential dilation. Photographs at 0 and 15 minutes.

pupillary function. 'Although anterior chamber depth is known to decline with age, ${ }^{7}$ in this study the relationship between pupil dilation ratio and anterior chamber depth had no correlation with the age of our subjects. Age therefore cannot account for the pupil dila-

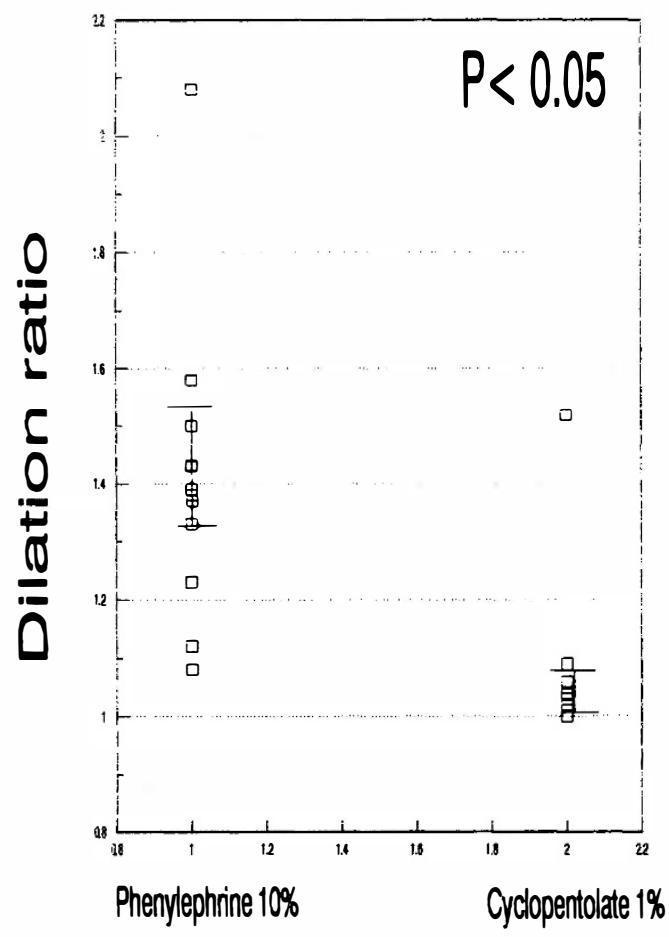

Fig. 4. Dilation ratio for patients with shallow anterior chambers. The anterior chamber depth has a significant effect on the dilation ratio after 15 minutes in eyes treated with Phenylephrine Hydrochloride $10 \%$ $(p<0.05)$. tion characteristics observed with Phenylephrine hydrochloride $10 \%$. Bienfang et al. ${ }^{8}$ showed that mydriatic applied locally on the limbus causes dilation to occur in that section of the pupil nearest the site of applicatiion. The vertical nature of the oval pupil could, therefore, be attributed to pooling of the mydriatic agent in the tear meniscus. Mechanical apposition of the iris to the anterior lens surface may vary in eyes with differing anterior chamber depths, ${ }^{2}$ possibly explaining different dilation characteristics. However, both of these factors, namely pooling of drug in the tear meniscus and mechanical apposition, should equally affect dilation caused by Phenylephrine hydrochloride $10 \%$ and Cyclopentolate hydrochloride $1 \%$, but this was not found to be the case in this study. Moreover, pooling in the tear meniscus would cause preferential dilation at the inferior pole unlike the preferential dilation at the superior and inferior poles observed in this study. Therefore, neither mechanical apposition of the iris and lens or drug pooling would explain vertical preferential dilation with Phenylephrine

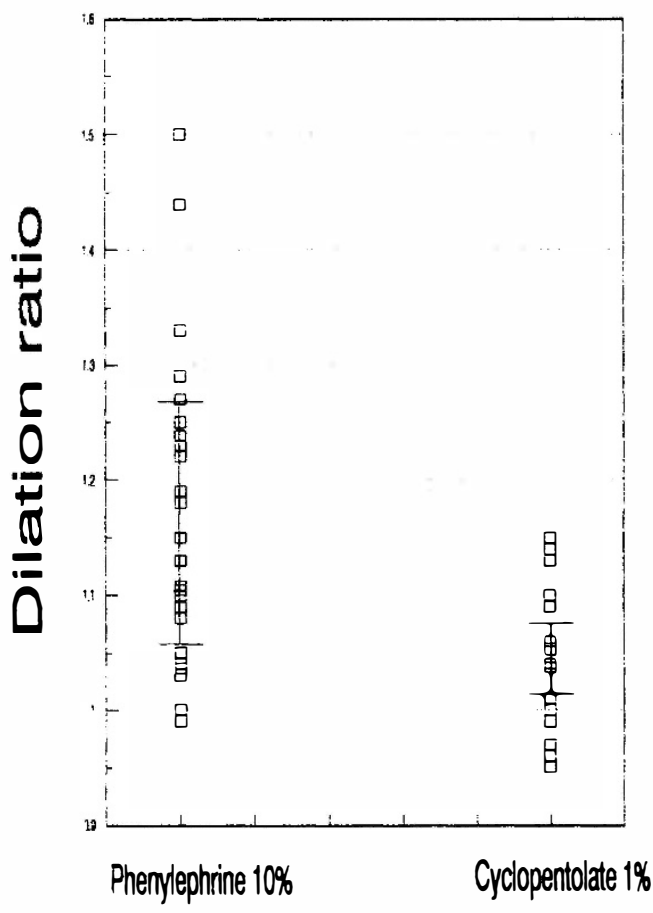

Fig. 5. Dilation ratio for patients with Deep anterior chambers. The anterior chamber depth has no significant effect on the dilation ratio after 1.5 minutes. 

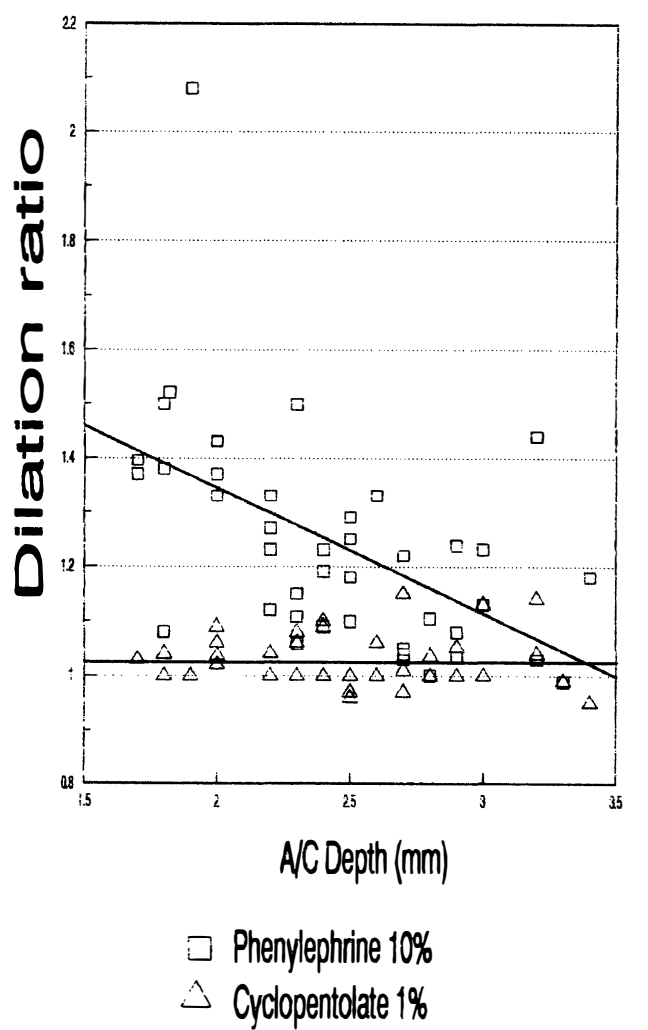

Fig. 6. A comparison of dilation ratios with anterior chamber depth. A correlation is show'n in eyes treated with Phenylephrine Hydrochloride $10 \%(r=0.526)$. No correlation was found in eyes treated with Cyclopentolate Hvdrochloride $1 \%(\dot{r}=0.002)$.

hydrochloride $10 \%$ and not with Cyclopentolate hydrochloride $1 \%$. The explanation may have an anatomical or physiological basis, either a variable sympathetic innervation with a higher intensity of receptors in the superior and inferior poles of the iris, or a preferential distribution of dilator muscle fibres in the vertical meridian. We are currently investigating these possibilities using immunocytochemistry and special staining for adrenergic receptors and smooth muscles.

Abnormal ocular autonomic function can predispose individuals to APACG by modification of physiological pupil block particularly in eyes with shallow anterior chambers. ${ }^{2}$
We have shown that sympathetic stimulation with Phenylephrine hydrochloride $10 \%$ can cause an oval pupil in eyes with a shallow anterior chamber. That a similar vertically oval pupil is usually seen in APACG supports the concept that aberrant sympathetic activity may be the cause of differential pupil dilation in the vertical meridian.

If the observed effect is due to an abnormal sympathetic innervation it could be part of the sympathetic abnormalities associated with APACG. The high dilation ratio may suggest an individual is at risk of APACG and could also increase the sensitivity of mydriatic testing in patients with shallow anterior chambers. Long term observation and controlled clinical studies will be needed to confirm this.

\section{Consent}

The results obtained for this manuscript were obtained after the nature of the procedure had been fully explained to the subjects and consent obtained.

Key words: Iris autonomic inervation, Pupil dilation, Acute glaucoma, Anterior chamber depth.

\section{References}

${ }^{\prime}$ Duke-Elder S and Jay B: Diseases of the lens and vitreous, glaucoma and hypotony. In Duke-Elder $\mathrm{S}$ ed. System of Ophthalmology, London, Henry Kimpton 1969: Volume XI: 569-71.

2 Barkan O: Glaucoma: classification, causes and surgical control. Am J Ophthalmol 1938, 21: 1099-1117.

'Philips: Closed Angle Glaucoma-Significance of sectoral variations in angle depth. BrJ Ophthalmol 1956. 40: 136-40.

${ }^{4}$ Thompson HS, Zackon DH, Czarnecki JSC: Tadpole shaped pupils caused by segmental spasm of the iris dilator muscle. Am J Ophthalmol 1983, 96: 467-477.

5 Smith RJH: A new method of estimating the depth of the anterior chamber. Br J Ophthalmol 1979 , 63: $215-20$.

'Dorczyn AD. Laor N, Nemet P: Sympathetic pupillary tone in old age. Arch Ophthalmol 1976, 94: 1905-6.

${ }^{7}$ Weekers R, Grieten J, Lavergne G: Study of the dimensions of the human anterior chamber. Ophthalmologica 1961, 142: 650 .

${ }^{8}$ Bienfang DC: Sector pupillary dilation with an epinephrine strip. Am J Ophthalmol 1973, 75: 883. 